\title{
ABUNDANCIA POBLACIONAL DE Tetraclita Panamensis (Cirripedia: Tetraclitidae) BAJO DOS DIFERENTES CONDICIONES DE OLEAJE EN ISLA PALMA, PACÍFICO COLOMBIANO
}

\section{POPULATION ABUNDANCE Tetraclita Panamensis (Cirripedia: Tetraclitidae) UNDER TWO DIFFERENT WAVE CONDITIONS IN PALMA ISLAND, COLOMBIAN PACIFIC}

\author{
Luis David Lizcano-Sandoval $^{1,}$, Sergio Iván Castro ${ }^{1}$ \\ ${ }^{1}$ Programa de Biología, Facultad de Ciencias Naturales, Universidad del Valle. \\ Recibido: Octubre 1 de 2011 \\ Aceptado: Noviembre 28 de 2011 \\ Correspondencia: Departamento de Biología, Universidad del Valle, Ciudad Universitaria Meléndez, Calle 13 No 100-00. Cali - Valle - \\ Colombia, correo electrónico: lizcanosandoval@gmail.com
}

\section{RESUMEN}

Las poblaciones de balanos como Tetraclita panamensis están sometidos a factores bióticos y abióticos verticales de la zona intermareal, y también son susceptibles a las condiciones horizontales como el oleaje. Este estudio se enfocó en evaluar la abundancia horizontal y distribución vertical de T. panamensis bajo condiciones de oleaje fuerte y calmado, y de temperatura en Isla Palma (Pacifico Colombiano) como una medida indirecta de su adaptación a dichas condiciones. Se realizaron 30 cuadrantes en la zona occidental y 34 cuadrantes en la zona oriental de manera sistemática, en los que se registraron 1239 y 1156 individuos respectivamente. Las poblaciones de ambos lados de la isla no presentaron diferencias significativas $(p=0,319)$, esto sugieren que las poblaciones de T. panamesis se encuentran adaptadas a las condiciones de oleaje, mientras que la distribución vertical puede estar influenciada principalmente por las condiciones físicas y biológicas del terreno que reducen los efectos de la temperatura y humedad. Aun faltan estudios que demuestren que el oleaje está afectando el asentamiento y reclutamiento de los balanos en Isla Palma, y también de alguna forma su distribución.

Palabras Clave: abundancia, adaptación, balanos, oleaje, temperatura.

\section{ABSTRACT}

The populations of barnacles as Tetraclita panamensis are subject to biotic and abiotic factors in the vertical intertidal zone, and are also susceptible to the horizontal conditions as the waves. This study focused on assessing the horizontal abundance and vertical distribution of T. panamensis under conditions of strong and calm waves, and temperature in Palma Island (Colombian Pacific) as an indirect measure of adaptation to these conditions. Thirty plots were conducted in the western side and 34 in the eastern, in a systematic way, in which there were 1239 and 1156 individuals respectively. Populations of both sides of the island did not differ significantly $(p=0.319)$; this suggests that populations of T. panamesis are adapted to wave conditions, while the vertical distribution may be influenced primarily by physical and biological conditions of the land that reduce the effects of temperature and humidity. Currently, there are not enough studies that show that the wave is affecting the settlement and recruitment of barnacles in Isla Palma, and also, in some way their distribution.

Key words: abundance, adaptation, barnacles, waves, temperature.

\section{INTRODUCCIÓN}

Las poblaciones de invertebrados marinos, que viven en zonas intermareales son vulnerables a los gradientes de humedad, tiempo de inmersión para la alimentación, y condiciones como la temperatura y la salinidad regulan la supervivencia, la duración del desarrollo larval y el reclutamiento en muchas especies de invertebrados marinos (1), al igual que la depredación y los tipos de oleaje. Pero la mayoría de los estudios se han enfocado en la variación intraespecifica en las características 
formas de vida en ambientes intermareales $(2,3,4)$ y poco a través de un gradiente horizontal en la exposición del oleaje $(5,6)$ y otros factores.

La habilidad de un organismo para resistir una fuerza puede depender de la dirección y velocidad en la que la fuerza es aplicada (7) y con respecto al oleaje, este por lo general suele tener velocidad suficiente como para imponer fuerzas hidrodinámicas sobre los individuos intermareales moldeando así su tamaño, forma y longevidad $(8,9)$. Este factor y muchos otros pueden conducir a que la abundancia poblacional de una especie este estrechamente correlacionada con su adaptación local, como en el estudio de Anderson (10).

El potencial para la adaptación y diferenciación genética entre poblaciones se puede dar cuando distintos hábitats y condiciones están presentes dentro del rango de distribución de una especie y estos son mediados por la selección natural (11). Se conoce por diversos estudios que la exposición a las olas afecta a los organismos directamente a través de mecanismos como la perturbación física (12), flujos de propágulos y nutrientes (13), sedimentación (14) e indirectamente modificando el comportamiento, las interacciones bióticas, el estrés a la temperatura y a la desecación $(15,16)$. Mientras que estudios como los de Barnes (17), Chan y Williams (18), y Berger et al. (6) demuestran que la demografía de algunos balanos esta determinada por factores como el estrés por calor o interacciones de condiciones ambientales, y en algunos casos por la presencia abundante de algas que interfieren en su alimentación.

A partir de estas observaciones se puede razonar que la abundancia de individuos en un ambiente dado es un reflejo directo de su capacidad adaptativa a condiciones locales como el oleaje (19). Por esta razón, el objetivo de este trabajo es evaluar la abundancia de T. panamensis bajo dos condiciones locales de oleajes diferentes, teniendo en cuenta un factor adicional como la temperatura.

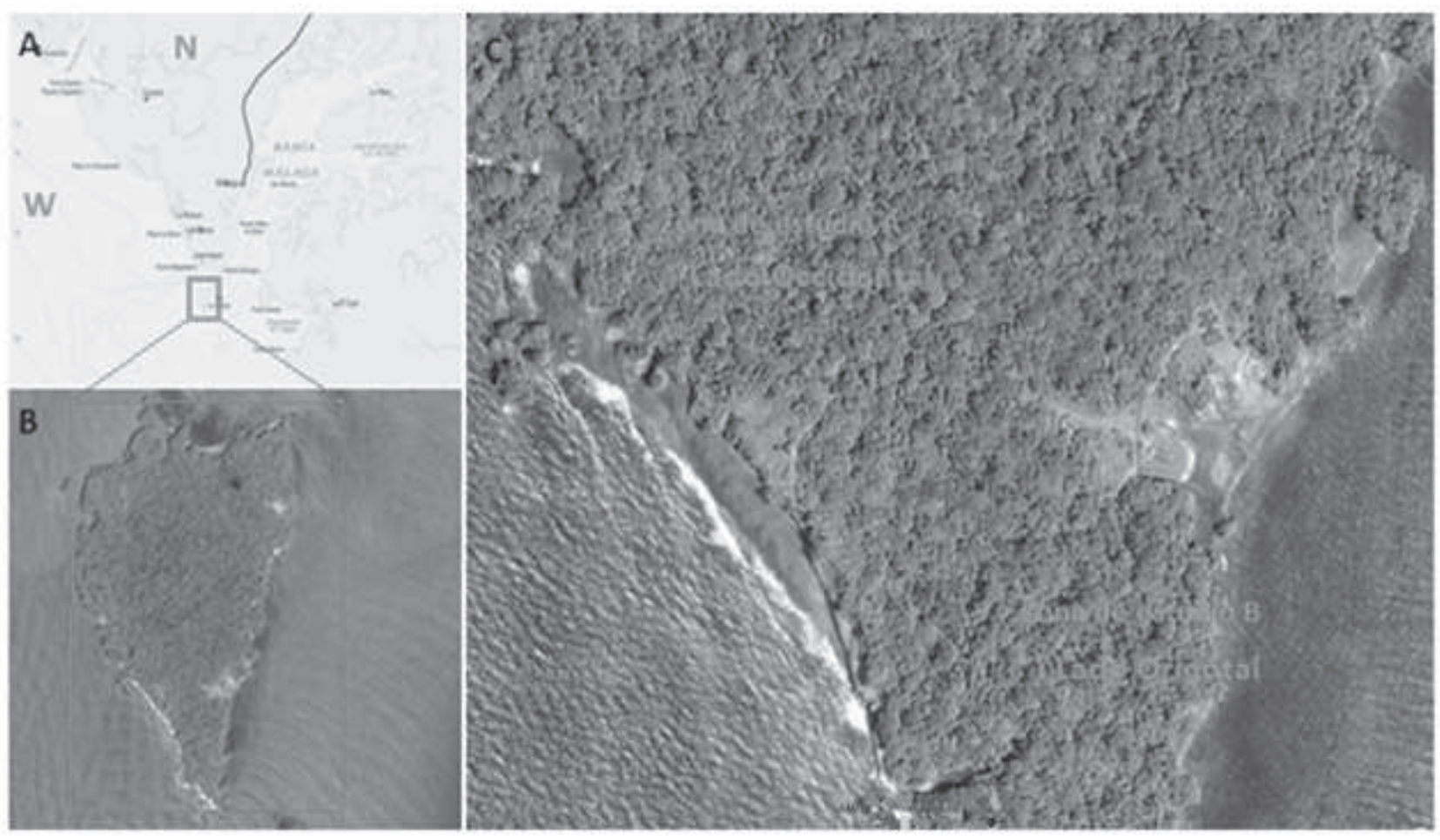

Figura 1. A) Posición geográfica de Isla de Palma (cuadro rojo) con respecto a Bahía Málaga, Pacifico colombiano. B) Vista aérea de Isla de Palma. C) Zonas de estudio dentro de la isla, a la izquierda, la zona occidental y a la derecha, la zona oriental. Nótese en los cuadros B y C el tipo de oleaje que recibe a cada zona. 


\section{MATERIALES Y MÉTODOS}

\section{Área de estudio}

El estudio se llevó a cabo en Isla de Palma $\left(3^{\circ} 54^{\prime} 24.68^{\prime \prime} \mathrm{N} ; 77^{\circ} 21^{\prime} 26.34^{\prime \prime O}\right)$ cerca de Bahía Málaga, Pacifico Colombiano (Figura 1A-B) durante el 16-21 de mayo del 2011. Dentro de esta isla, se seleccionaron dos zonas caracterizadas por sus diferentes tipos de oleaje. La zona ubicada en la cara occidental de la isla, se caracterizaba por un fuerte oleaje, mientras que la otra zona, ubicada en la cara oriental de la isla, se caracterizaba por un oleaje más calmado (Figura 1C).

\section{Toma de datos}

El muestreo se llevó a cabo por medio de un cuadrante de $40 \times 40 \mathrm{~cm}$ dispuesto de forma sistemática cada $3 \mathrm{~m}$ de distancia uno del otro a lo largo de la zona intermareal y con una altura entre 2,8-3,7 m con respecto a la marea baja de ambas zonas de estudio. El número total de Tetraclita panamensis contados dentro de los cuadrantes fue registrado. También se registró la altura máxima y mínima en la cual se halló un individuo para ambas zonas de estudio. Se tomaron datos de temperatura $\left({ }^{\circ} \mathrm{C}\right)$ del sustrato y de un individuo al azar dentro de cada cuadrante con un termómetro infrarrojo Horiba IT-540. La frecuencia del oleaje (olas $\mathrm{min}^{-1}$ ) se midió durante la marea baja entre las 1700 - 1800 horas del día 21 de mayo, y la velocidad del oleaje $\left(\mathrm{m} \mathrm{s}^{-1}\right)$ por medio de boyas distanciadas entre sí $10 \mathrm{~m}$, y a 19,5 m del acantilado occidental, mientras que en el oriental se distanciaron entre sí $6,2 \mathrm{~m}$, y a $11,2 \mathrm{~m}$ del acantilado.

\section{Análisis de datos}

Los datos se analizaron con una prueba $U$ de MannWhitney. La hipótesis científica nula probada fue que no habían diferencias significativas entre la abundancia poblacional de T. panamensis en las dos zonas de estudio. También se evaluó el efecto de la temperatura con un ANOVA de una vía, y las comparaciones a posteriori con la prueba de Tukey. Por último, se compararon las frecuencias y las velocidades del oleaje de ambas zonas de estudio con una prueba de t-student. Todos los análisis estadísticos se realizaron con un nivel de confianza del 95\%. Los análisis se llevaron a cabo con el software estadístico StatGraphics Plus v5.1.

\section{RESULTADOS}

En total se obtuvieron 30 cuadrantes en la zona occidental y 34 cuadrantes en la zona oriental, en los que se registraron 1239 y 1156 individuos de Tetraclita panamensis respectivamente, y se obtuvieron medias muestréales muy variables para las dos zonas, en el lado occidental se obtuvo 41,3 $\pm 65,5$ individuos y para el lado oriental $34 \pm 54,3$ individuos. El número máximo de individuos contados en un cuadrante fueron 315 y 204 para la zona occidental y oriental, respectivamente. Durante el muestreo se encontró que la altura de distribución intermareal de T. panamensis era diferente en las dos zonas. El lado occidental se hallaron individuos entre $2,8-3,9 \mathrm{~m}$ de altura con respecto a la marea baja mínima, mientras que en el lado oriental la distribución fue entre 1,56-3,36 m de altura.

Con respecto a las abundancias, los conjuntos de datos en las cajas de la zona occidental y zona oriental son similares, de los cuales solo se observó un dato atípico (Figura 2). La prueba de MannWhitney no reveló diferencias significativas entre la abundancias poblacionales de T. panamensis en las dos zonas de estudio para un nivel de confianza del $95 \%(p=0,319)$.

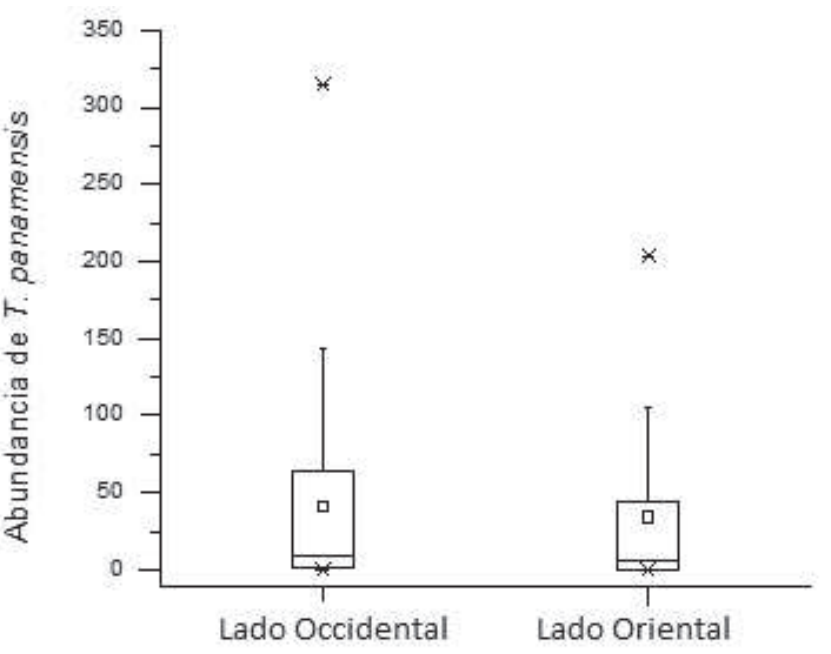

Figura 2. Diagrama de cajas para la abundancia de $T$. panamensis en las zonas A y $\mathrm{B}$. 
También se observó que los balanos se fijaban sobre sustrato rocoso (limolita) sin poros o agujeros como los usados por los cangrejos Pachygrapsus transversus. Ambas zonas presentaron sitios de sombra, luz intermedia o luz directa y corrientes de agua dulce. A diferencia del lado occidental, en el lado oriental se presenció follaje a orilla del acantilado (Figura 3, A y B).

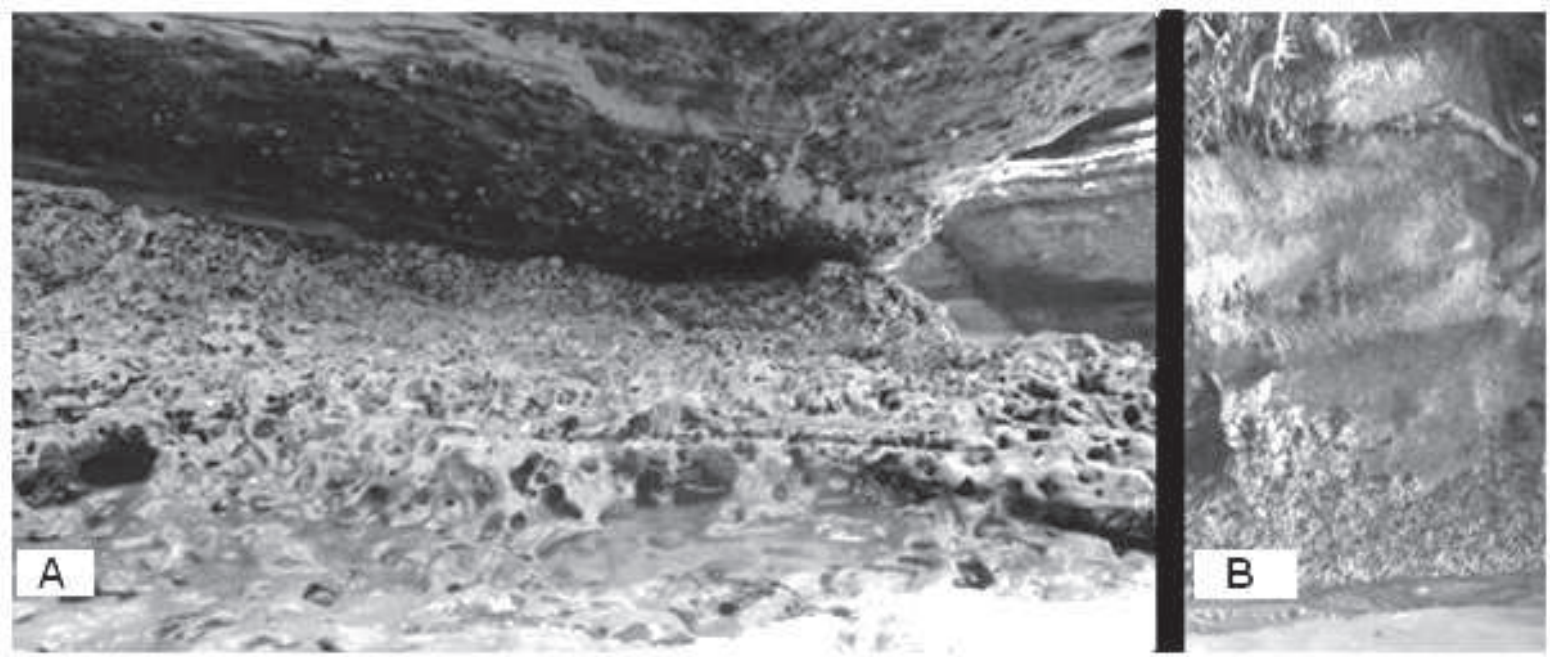

Figura 3. A) Foto tomada en el lado occidental donde se puede observar a T. panamensis creciendo por dentro de una grieta horizontal en la parte superior. B) Foto tomada en el lado oriental donde se puede observar las el patrón del terreno vertical, desde abajo hasta arriba: suelo arenoso, piedra degrada, piedra lisa homogénea, estrato de algas y raíces de arboles que crecen en el borde.

Las temperaturas en ambas zonas de estudio estuvieron entre $26,7-38^{\circ} \mathrm{C}$ (Tabla 1 ), y se encontraron diferencias significativas entre las temperaturas de los balanos y los sustratos en ambas zonas ( $p=0,033$, ANOVA una vía), siendo la comparación entre la temperatura de los balanos de la zona occidental y la zona oriental la única con diferencias significativas ( $p=0,026$, Tukey).

Tabla 1. Estadística descriptiva de los datos de temperatura $\left({ }^{\circ} \mathrm{C}\right)$ de sustrato y del balano en cada cuadrante de ambas zonas estudiadas.

\begin{tabular}{lllcccc}
\hline & & $\mathrm{N}$ & $\overline{\mathrm{X}}$ & $\mathrm{DE}$ & $\mathrm{Min}$ & $\mathrm{Max}$ \\
\hline Zona & Sustrato & 30 & 28,5 & $\pm 2,07$ & 26,7 & 34 \\
occidental & Balano & $24 *$ & 27,8 & $\pm 1,60$ & 26,7 & 34 \\
Zona & Sustrato & 34 & 29,6 & $\pm 2,79$ & 26,7 & 38 \\
oriental & Balano & $23 *$ & 29,1 & $\pm 2,26$ & 26,7 & 34 \\
\hline
\end{tabular}

*se asume esta cantidad debido a que no hubo balanos en todos los cuadrantes.

Las frecuencias del oleaje (olas $\mathrm{min}^{-1}$ ) en ambas zonas no presentaron diferencias significativas entre sí (estadístico $t=1,25 \mathrm{E}-15, p>0,999$ ), al igual que las velocidades del oleaje de ambas zonas (estadístico $t=$ $0,314, p=0,757)$. El promedio de olas en las ambas zonas fue de 3,50 olas $\mathrm{min}^{-1}$, mientras que la velocidad estuvo alrededor de los 3,50 $\mathrm{m} \mathrm{s}^{-1}$ (Tabla 2).
Tabla 2. Estadística descriptiva de los registros de frecuencias (olas $\mathrm{min}^{-1}$ ) y velocidades $\left(\mathrm{m} \mathrm{s}^{-1}\right)$ del oleaje en ambas zonas de estudio.

\begin{tabular}{llccccc}
\hline & & $\mathrm{N}$ & $\overline{\mathrm{X}}$ & $\mathrm{DE}$ & $\mathrm{Min}$ & $\mathrm{Max}$ \\
\hline Zona & Frecuencia Olas & 20 & 3,50 & $\pm 1,15$ & 1,0 & 6,0 \\
Occidental & Velocidad Olas & 10 & 3,45 & $\pm 0,81$ & 2,5 & 4,8 \\
Zona & Frecuencia Olas & 20 & 3,50 & $\pm 1,10$ & 1,0 & 6,0 \\
Oriental & Velocidad Olas & 10 & 3,56 & $\pm 0,70$ & 2,5 & 5,3 \\
\hline
\end{tabular}

\section{DISCUSIÓN}

La abundancia de los balanos bajo condiciones de oleaje diferentes en Isla Palma no fue significativamente diferente como se esperaba. Aunque los resultados indican que no hay diferencias en la frecuencia y velocidad del oleaje, no significa que el impacto de las olas sobre la costa rocosa intermareal sean similares en ambos lados, ya que, otro posible índice, el cual no se evaluó, es el peso de la ola (9), que puede ser utilizado para derivar velocidades de flujo, y puede explicar la fuerza con que las olas impactan en la zona occidental. Las medidas indirectas más 
comunes del peso de las olas se obtienen por la medición de la morfología costera local e información de la velocidad del viento y su dirección $(20,21)$.

Las características del área de estudio en Isla Palma no son las mas adecuadas para que exista un patrón de corriente en la marea que pueda retener las larvas de los balanos y mantener así las poblaciones aisladas (Figura 1C), como lo encontró Dewolf (22) en otro estudio. Además, el periodo larval característico de los balanos puede tardar entre ocho y quince días (23), lo que es suficiente para que las larvas puedan llegar a la costa oriental de la isla desde de la costa occidental. Aunque algunos autores hayan registrado mortalidades, en otras áreas, que varían dramáticamente sobre una escala espacial relativamente pequeña (24 - 26) es posible que esta mortalidad tampoco sea suficiente para producir diferenciación, ya que según Brown et al. (27), el flujo génico entre dos poblaciones por medio de polimorfismos de longitud de fragmentos de restricción (RFLP) de la región D-loop mitocondrial, a lo largo de una región de más de $30 \mathrm{Km}$ de distancia, las poblaciones se comportan como una sola población panmíctica. Además se debe tener en cuenta la dispersión de una larva de balano puede ser de hasta $100 \mathrm{Km}$ (28).

Las diferencias obtenidas en temperatura y distribución de los balanos en ambas zonas muestran una inconsistencia con lo esperado ya que en la zona oriental, la cual tiene mayor temperatura, presenta una distribución de $T$. panamensis dentro de un espectro mayor en comparación a la población de la zona occidental. Pero estas diferencias pueden ser debidas a las características de los terrenos ya que en la zona occidental se pudo observar que la distribución esta restringida a las grietas horizontales que se forman (Figura 3A), mientras que en la zona oriental el acantilado se caracterizaba por presentarse en forma de una pared vertical homogénea, sin grietas, y con bastante presencia de algas y arboles en la orilla (Figura 3B), lo cual podía permitir que los balanos asentaran a alturas más bajas por estar protegidos en la sombra prácticamente en la mayoría del tiempo. Específicamente, la parte superior del límite intermareal no fue notablemente diferente como la parte inferior. Igualmente se conoce que el calor y las condiciones de sequia son los principales factores en la mortalidad de individuos postasentados en los limites superiores de la zona intermareal (18), pero es posible que los individuos aquí estudiados no estén muy influenciados por estos factores debido a que generalmente estos eran hallados bajo sombra y que además estas zonas eran bañadas por pequeñas corrientes de agua dulce provenientes de la isla.

Dentro del sistema intermareal superior, el balance entre los grupos de organismos puede ser alterado como resultado de procesos ecológicos como la competencia, depredación y perturbación $(29,30)$, además, hay evidencia de que estos factores pueden aportar a que hayan diferencias de abundancia de organismos entre áreas intermareales de aguas turbulentas y aguas calmadas (15), pero en el trabajo de Cuellar (31), en zonas intermareales de Isla Palma (Pacifico Colombiano) no se encontraron diferencias en la abundancia de dos especies de balanos, Tetraclita panamensis y Chthamalus sp., por ausenciapresencia de su depredador primario el gasterópodo Thais melones, por lo tanto, sugerimos que la poca diferencia en la distribución superior intermareal en las dos zonas de estudio, puede deberse a otro tipo de interacciones bióticas, en las que las algas tendrían un papel importante, y que involucran también factores abióticos, como lo evidencia la comparación de las figuras $3 \mathrm{~A}$ y $3 \mathrm{~B}$, y estudios realizados que han demostrado que la demografía de algunos balanos está determinada por la presencia abundante de algas que interfieren en su alimentación $(17,18,6)$.

Thiyagarajan et al. (32) sugieren que aun falta una comprensión más detallada del desarrollo de las larvas y los patrones de fijación que se requieren para evaluar los efectos de factores ambientales 
sobre estas y su contribución a la dinámica de la población, además teniendo en cuenta que el asentamiento y el reclutamiento son procesos muy diferentes y no necesariamente están relacionados estrechamente. El asentamiento se da en larvas de vida libre y puede involucrar la selección de hábitat, mientras que el reclutamiento, es la supervivencia de los juveniles asentados (33). Tambien, Moore (34) y Hatton (35) encontraron que la densidad del asentamiento y el crecimiento de dos especies de balanos fue menor en aguas calmadas, y Connell (15) afirma que algunas diferencias en la abundancia entre aguas turbulentas y aguas mas calmadas puede deberse a interacciones biológicas muy complicadas.

Los balanos T. panamensis parecen estar bien adaptados a las condiciones locales de oleaje, debido a que su abundancia no presenta diferencias en ambas zonas estudiadas en Isla Palma. Su distribución, por el contrario, si difiere y uno de los factores que esta afectando esta distribución puede ser la temperatura y las características físicas de cada zona estudiada. Aun faltan estudios que demuestren como el oleaje esta afectando el asentamiento y reclutamiento de los balanos en Isla Palma, y también de alguna forma su distribución. Aunque existe la posibilidad de que las poblaciones puedan ser modificadas genéticamente por una mortalidad debida a aspectos selectivos de la localidad en algunas características fenotípicas dadas (36; Drouin et al, 2002), es necesario otros estudios que apoyen esta premisa, ya que las posibles diferencias genéticas entre las dos poblaciones no fueron manifestadas por la variación en la abundancia en función del tipo de oleaje.

\section{BIBLIOGRAFÍA}

1. Pechenik, J. A. (1987). "Environmental influences on larval survival and development". En A. C. Giese, J. S. Pearse y V. B. Pearse, (Eds.), Reproduction of Marine Invertebrates (551-608). Palo Alto: Blackwell.

2. Gosselin, L. y Qian, P. (1997). “Juvenile mortality in benthic marine invertebrates". En Marine Ecology Progress Series, 146, 265-282.

3. Benedetti-Cecchi, L., Acunto, S., Bulleri, F. y Cinelli, F. (2000). "Population ecology of the barnacle Chthamalus stellatus in the northwest Mediterranean". En Marine Ecology Progress Series, 198, 157-170.

4. Phillips, N. E. (2002). "Effects of nutrition-mediated larval condition on juvenile performance in a marine mussel". En Ecology, 83, 2562-2574.

5. Etter, R. J. (1989). "Life history variation in the intertidal snail Nucella lapillus across a wave-exposure gradient". En Ecology, 70, 1857-1876.

6. Berger S. M., Darrah, A. J. y Emlet, R. B. (2006). "Spatial and temporal varaibility of early post-settlement survivorship and growth in the barnacle Balanus glandula along an estuarine gradient". En Journal of Experimental Marine Biology and Ecology, 336, 74-87

7. Grenon, J. y Walker, G. (1981). "The tenacity of the limpet Patella vulgata L.: An experimental approach". En Journal of Experimental Marine Biology and Ecology, 54, 277-308.

8. Denny, M. W., Daniel, T. y Koehl, M. (1985). "Mechanical limits to size in wave-swept organisms". En Ecological Monographs, 55, 69-102.

9. Denny, M. W. (1988). Biology and the mechanics of the wave-swept environment. Princeton: Princeton University Press. 
10. Anderson, D. T. (1994). Barnacles: Structure, Function, Development and Evolution. London: Chapman and Hall.

11. Schmidt, P. y Rand, D. (1999). "Intertidal microhabitat and selection at MPI: interlocus contrasts in the northern acorn barnacle Semibalanus balanoides". En Evolution, 1, 135-146.

12. Vadas, R., Wright, W. y Mille, L. (1990). "Recruitment of Ascophyllum nodosum: wave action as a source of mortality". En Marine Ecology Progress Series, 61, 263-272.

13. Bertness, M., Gaines, S., Stephens, E.G. y Yund, P. (1992). "Components of recruitment in populations of the acorn barnacle Semibalanus balanoides (Linnaeus)". En Journal of Experimental Marine Biology and Ecology, $156,199-215$.

14. Airoldi, I. y Cinelli, E. (1997). "Effects of sedimentation on subtidal macroalgal assemblages: an experimental study from a mediterranean rocky shore". En Journal of Experimental Marine Biology and Ecology, 215, 269288.

15. Connell, J. H. (1972). "Community interactions on marine rocky intertidal shores". En Annual Review of Ecology and Systematics, 3, 169-192.

16. Menge, B. A. (1976). "Organization of the New England rocky intertidal community: role of predation, competition, and environmental heterogeneity". En Ecological Monographs, 46, 355-393.

17. Barnes, H. (1956). "Balanus balanoides (L.) in the Firth of Clyde: the development and annual variation of the larval population and the causative factors". En Journal of Animal Ecology, 25, 72-84.

18. Chan, B. y Williams, G. (2003). "The impact of physical stress and molluscan grazing on the settlement and recruitment of Tetraclita species (Cirripedia: Balanomorpha) on a tropical shore". En Journal of Experimental Marine Biology and Ecology, 284, 1-23.

19. Crisp, D. y Maclean, F. (1990). "The relation between the dimension of the cirral net, the beat frequency and the size and of the animal in Balanus balanoides and Elminius modestus". En Journal of Marine Biology Association of the UK, 70, 505-513.

20. Thomas, M. L. (1986). "A physically derived exposure index for marine shorelines”. En Ophelia, 25, 1-13.

21. Ruuskanen, A., Bick, S. y Reitalu, T. (1999). "A comparison of two cartographic exposure methods using Fucus vesiculosus as an indicator". En Marine Biology, 134, 139-145.

22. Dewolf, P. (1973). "Ecological observations on the mechanisms of dispersal of barnacle larvae during planktonic life and settling". En Netherlands Journal of Sea Research, 6, 1-129.

23. Strathmann, R., Branscomb, E. y Veder, K. (1981). "Fatal errors in set as a cost of dispersal and the influence of intertidal flora on set of barnacles". En Oecologia, 48, 13-18.

24. Young, A. M. y Chia, F. S. (1984). "Microhabitat-associated variability in survival and growth of subtidal solitary ascidians during the first 21 days after settlement". En Marine Biology, 81, 61-68.

25. Wethey, D. S. (1986). "Local and regional variation in settlement and survival in the littoral barnacle Semibalanus balanoides (L.): patterns and consequences". En P. Morre y R. Seed (Eds.), The Ecology of Rocky Coasts (194-202). New York: Columbia University Press. 
26. Jarrett, J. N. (2000). "Temporal variation in early mortality of an intertidal barnacle". En Marine Ecological Progress Series, 204, 305-308.

27. Brown, A. F., Kann, L. M. y Rand, D. M. (2001). "Gene flow versus local adaptation in the nortern acorn barnacle Semibalanus balanoides: Insights from mitochondrial DNA variation". En Evolution, 55, 1972-1979.

28. Flowerdew, M. W. (1983). "Electrophoretic investigation of populations of the cirrepede Balanus balanoides (L.) around the North Atlantic seaboard". En Crustaceana, 45, 260-278.

29. Connell, J. H. (1975). "Some mechanisms producing structure in natural communities: a model and evidence from field experiments". En M. L. Cody y J. M. Diamond (Eds.), Ecology and evolution of communities (4604940). Cambridge: Belknap Press.

30. Bosman, A., Hockey, P. y Sigfried, W. (1987). "The influence of coastal upwelling on the functional structure of rocky intertidal communities". En Oecologia, 72, 226-232.

31. Cuellar, J. L. (2001). "Relación entre la abundancia de tres posibles presas (balanos, lapas y neritas) y la distribución de Thais melones (Gasteropoda: Muricidae) en un acantilado intermareal de Isla Palma, Pacifico colombiano". Tesis de pregrado. Departamento de Biología. Universidad del Valle, Cali. 41 p.

32. Thiyagarajan, V., Harder, T. y Qian, P. Y. (2003). "Combined effects of temperatura and salinity on larval development and attachment of the subtidal barnacle Balanus trigonus Darwin". En Journal of Experimental Marine Biology and Ecology, 287, 223-236.

33. Caffey, H. M. (1985). "Spatial and temporal variation in settlement and recruitment of intertidal barnacles". En Ecological Monographs, 55, 313-332.

34. Moore, H. B. (1935). "The biology of Balanus balanoides IV. Relation to environmental factors". En Journal of Marine Biology Association of the UK, 20, 279-307.

35. Hatton, H. (1938). "Essais de bionomie explicative sur quelques especes intercotidales d'algues et d'animaux". En Annales de I'Institut Oceanographique Monaco, 17, 241-348.

36. Brind'amour, A., Bourget, E. y Tremblay, R. (2002). "Fecundity, growth rate and survivorship at the interface between two contiguous genetically distinct groups of Semibalanus balanoides". En Marine Ecology Progress Series, 229, 173-184.

37. Drouin, C.-A., Bourget, E. \& Tremblay, R. (2002). "Larval transport processes of barnacle larvae in the vicinity of the interface between two genetically different populations of Semibalanus balanoides". En Marine Ecology Progress Series, 229, 165-172. 